\title{
Dry Eye, CTCAE
}

National Cancer Institute

\section{Source}

National Cancer Institute. Dry Eye, CT CAE. NCI Thesaurus. Code C143410.

A disorder characterized by dryness of the cornea and conjunctiva. 